\title{
COMORBILIDAD ENTRE EL DISTRÉS PSICOLÓGICO Y ABUSO/ DEPENDENCIA DE DROGAS, CIUDAD DE GUATEMALA - GUATEMALA
}

\author{
Miriam García Estrada ${ }^{1}$, Robert Mann², Carol Strike ${ }^{3}$,Bruna Brands ${ }^{4}$, Akwatu Khenti ${ }^{5}$
}

\begin{abstract}
${ }^{1}$ Ph.D. Profesora Titular de la Facultad de Ciencias Medicas. Universidad de San Carlos de Guatemala, Guatemala. E-mail: andragogiamiriam@yahoo.com

2 Ph.D. Investigator. Centre for Addiction and Mental Health, University of Toronto, Canada. E-mail: robert_mann@camh.net

${ }^{3}$ Ph.D. Investigator. Health Systems Research and Consulting Unit. Centre for Addiction and Mental Health. University of Toronto, Canada. E-mail: carol_strike@camh.net

${ }^{4}$ Ph.D. Investigator. Office of Research and Surveillance, Drug Strategy and Controlled Substances Program. Health Canada and Public Health and Regulatory Policies. Center for Addiction and Mental Health. University of Toronto, Canada. E-mail: bruna_brands@camh.net

${ }^{5}$ MSc. Director Office International Health. Assistant Professor at University of Toronto. Dalla Lana School of Public Health. Canada. E-mail: akwatu_khenti@camh.net
\end{abstract}

RESUMEN: El objetivo del estudio fue investigar la prevalencia de distrés psicológico en pacientes atendidos en centros especializados en alcohol y drogas en la Ciudad de Guatemala. Es un estudio transversal con 91 pacientes mayores de 18 años atendidos en el Centro de Tratamiento de la Secretaria Ejecutiva de la Comisión contra las Adicciones y el Trafico Ilícito de Drogas, y en Casa Hogar Jireh. Se aplicó un cuestionario adaptado del formulario de la EULAC-CICAD para caracterizar a los participantes. La escala de Kessler-10 se aplico para detención de distrés psicológico. Los resultados muestran que 68\% de la muestra total tenían entre 18 y 39 años, la edad promedio es 44. El 95.6\% eran hombres. Refieren diagnostico previo (32.9\%), relacionado con ansiedad en 2.4\%, depresión en 3.3\%, esquizofrenia en $4.4 \%$, y trastorno bipolar en $4.4 \%$. Presentan diagnostico actual de salud mental 17.58\%. Conclui-se que El K-10 indica que $31.87 \%$ tienen niveles severos el 39.56\% muy severo de distrés psicológico (71.43\%). Datos sensibles a ansiedad y depresión, son similares a los reportados en otros estudios internacionales.

DESCRIPTORES: Comorbilidad. Distrés psicológico. Trastornos mentales. Trastornos por uso de sustancias.

\section{COMORBIDITY OF PSYCHOLOGICAL DISTRESS AND ABUSE/ DEPENDENCE OF DRUGS, GUATEMALA CITY - GUATEMALA}

\begin{abstract}
The aim of this study was to investigate the prevalence of psychological distress in patients treated at centers specialized in alcohol and drugs in Guatemala City. Cross-sectional study with 91 patients older than 18 years treated at the Treatment Center Executive Secretary of the Commission against Addictions and Illicit Drug Trafficking, and Casa Hogar Jireh. A questionnaire was adapted from form EULAC-CICAD to characterize the participants. The Kessler-10 scale was applied for detention of psychological distress. The results show that $68 \%$ of the total sample were between 18 and 39 years, the average age is $44.95 .6 \%$ were men. $32.9 \%$ referred previous diagnosis, related to anxiety $(2.4 \%)$, depression $(3.3 \%)$, schizophrenia $(4.4 \%)$, and bipolar disorder $(4.4 \%)$. The current mental health diagnosis is $17.58 \%$. Conclusion: The K-10 indicates that $31.87 \%$ had severe and $39.56 \%$ very severe levels of psychological distress. This study shows that $71.43 \%$ is sensitive data with anxiety and depression and are similar to those reported in other international studies.
\end{abstract}

DESCRIPTORS: Comorbidity. Substance use-related disorders. Psychological distress. Mental health assistance.

\section{COMORBIDADE ENTRE SOFRIMENTO PSÍQUICO E ABUSO/ DEPENDÊNCIA DE DROGAS, CIDADE DE GUATEMALA - GUATEMALA}

RESUMO: O objetivo deste estudo foi investigar a prevalência de sofrimento psíquico em pacientes tratados em centros de álcool e drogas na Cidade da Guatemala. Estudo transversal com 91 pacientes acima de 18 anos atendidos no Centro de Tratamento de Secretário Executivo da Comissão contra Vícios e Tráfico Ilícito de Drogas, Jireh e Casa Hogar. Um questionário foi adaptado de forma EULAC-CICAD para caracterizar os participantes. A escala de Kessler-10 foi aplicada para a detenção de sofrimento psíquico. Os resultados mostram que $68 \%$ do total da amostra tinham entre 18 e 39 anos, com idade média de $44.95,6 \%$ eram homens. 32,9\% referiram diagnóstico prévio, relacionado à ansiedade (2,4\%), depressão (3,3\%), esquizofrenia $(4,4 \%)$ e transtorno bipolar $(4,4 \%)$. 17,58\% têm o diagnóstico de saúde mental atual. Conclui-se que o K-10 indica que 31,87\% tinham níveis de 39,56\% de sofrimento psíquico grave $(71,43 \%)$. Dados confidenciais com ansiedade e depressão são semelhantes aos relatados em outros estudos internacionais.

DESCRITORES: Comorbidade. Sofrimento psíquico. Transtornos relacionados ao uso de substâncias. Assistência em saúde mental. 


\section{INTRODUCCIÓN}

La salud mental es determinante en la calidad de vida de las poblaciones. Los problemas de salud mental afectan a la sociedad en general ${ }^{1} \mathrm{y}$ no solo a algunos sectores de la misma, por tanto representan un desafío para alcanzar el desarrollo general. No existe un grupo humano inmune a ellos, pero es evidente que el riesgo es mayor en los pobres, marginados, desempleados, personas con poco nivel de escolaridad, victimas de violencia, migrantes y refugiados, indígenas, mujeres y niños maltratados y la población anciana desatendida. El riesgo de consumo de drogas en pacientes con trastornos mentales es alto así como la presencia de trastornos mentales derivados del uso de drogas licitas e ilícitas. La presencia de trastornos mentales y de uso de sustancias simultáneamente se conoce como comorbilidad y afecta la calidad de vida de las personas que los padecen y representa cargas emocionales y financieras tanto a la familia, la sociedad y los países generando disminución en la productividad para el desarrollo. ${ }^{2}$

La comorbilidad de trastornos por uso de sustancias con otros trastornos mentales es frecuente. La magnitud y carga del problema es grande pues 450 millones de personas sufren de un trastorno mental o de la conducta, cuatro de las seis causas principales de los años vividos en discapacidad resultan de trastornos neuropsiquiátrico (depresión, trastornos generalizados por el uso de alcohol y otras drogas, esquizofrenia y trastorno bipolar.) En América Latina, los problemas de salud mental no se conocen a profundidad y se presentan entremezclados como sufrimientos psicosociales colectivos, derivados no solo de los problemas específicos de la enfermedad mental sino de problemas estructurales de cada país. La pobreza, la violencia, el desempleo, desastres naturales, migraciones, guerras, aspectos culturales, dinámicas familiares entre otros resultan ser problemas prioritarios a atender por los sistemas de salud de cada país. ${ }^{3}$ Las propiedades especificas de las drogas (tipo de droga consumida, edad de inicio, vía de consumo) contribuyen a la aparición de un patrón de consumo que influyen en la presencia de la comorbilidad y deben estudiarse para establecer las necesidades de atención que debe darse dentro del ámbito de la salud mental a la población. Diversos estudios se han realizado en Estados Unidos, Australia, Canadá y Europa. ${ }^{4}$ Sin embargo para America Latina existen pocas investigaciones por lo que los datos que este estudio reporte servirán de base a otras investigaciones.
En el ámbito de la salud mental la relación de los trastornos por uso de sustancias esta bien documentado en países de otras regiones. Según El General Surgeon Report de salud mental de los Estados Unidos de 2006 la comorbilidad afecta a millones de personas cada año alredor de la mitad de los individuos diagnosticados con abuso o dependencia de alcohol u otras drogas también tienen un diagnóstico psiquiátrico: $26 \%$ tienen trastornos del estado de ánimo, 28\%, trastornos de ansiedad, $18 \%$, personalidad antisocial y $7 \%$, esquizofrenia. La presencia de depresión mayor entre los que usan drogas varía entre $30 \%$ a $50 \%$. Hallazgos y conclusiones similares se encuentran en diversas publicaciones. ${ }^{1-3}$ Los resultados de estudios como estos evidencian que son problemas cada vez mayores y la brecha de tratamiento es grande. ${ }^{4}$

Los criterios para clasificarlos son cada vez más completos así como las opciones de tratamiento. En octubre del 2008, la OMS presentó el Programa de Acción para superar las brechas en salud mental, mejora y ampliación de la atención de los trastornos mentales, neurológicos y por abuso de sustancias (mhGAP, por sus siglas en inglés). ${ }^{5}$ Este programa se basa en las mejores pruebas científicas disponibles y ofrece un conjunto de estrategias y actividades para ampliar y mejorar la atención de las personas con trastornos mentales, neurológicos y por abuso de sustancias.

En América Latina el abordaje de estos problemas implican profundizar en investigaciones que respondan a las condiciones políticas, sociales, culturas y étnicas de la región. Guatemala reporta estudios respaldados por la Secretaria Ejecutiva Comisión Contra las Adicciones y el Tráfico ilícito de Drogas (SECCATID) así también algunas tesis universitarias presentan datos del comportamiento de este fenómeno. Según el informe de la Junta Internacional para la Fiscalización de Estupefacientes, JIFE-ONU $2003^{6}$ el corredor de Centroamérica, México y el Caribe es una vía para el tráfico de cocaína y heroína desde Colombia a América del Norte. Desde enero de 2009 se está implementando la Política Nacional contra las Adicciones y el Tráfico Ilícito de Drogas. Esto refleja la búsqueda continua de la adaptación de la política interna a los acuerdos firmados por el país en la Convención de las Naciones Unidas en materia de drogas en 1961, renovado en la década de 1970. En 1994, un acuerdo gubernamental nacional fue promulgado por SECCATID. 
Como resultado de la existencia del corredor de tráfico de drogas, la disponibilidad de drogas en el país ha ido creciendo año tras año. El gobierno de Guatemala, a través de la SECCATID, está trabajando arduamente para aplicar las medidas de control dirigidas a reducir el uso y abuso de drogas lícitas e ilícitas en las escuelas, universidades, cárceles y sectores específicos como el barrio "El Gallito", el más conocido lugar donde se venden drogas en Ciudad de Guatemala.

En Guatemala el Ministerio de Salud Pública y Asistencia Social es el encargado de evaluar la calidad de los servicios e instituciones que ofrecen programas de tratamiento de drogas. Este trabajo se realiza a través del Departamento de Regulación, Acreditación y Control de Establecimientos de Salud (DRACES). Según el Mecanismo de Evaluación Multilateral ${ }^{7}$, Guatemala cuenta con 92 centros de tratamiento, aunque por la demanda, operan cerca de 100 que esperan la acreditación.

La Organización Mundial de Salud estimaba que en Guatemala la prevalencia de los principales trastornos de salud mentales, TSM, era: depresión (25.5\%), psicosis crónica $(21.9 \%)$, epilepsia (10.4\%), trastorno bipolar (5.2\%), trastornos somatomorfos $(11.4 \%)$, retraso mental $(4.4 \%)$ $\mathrm{y}$ trastornos de ansiedad (8.3\%). ${ }^{8}$ Algunos hechos históricos específicos de Guatemala han suscitado especial interés para la investigación, como los determinantes culturales del consumo de alcohol entre la población maya ${ }^{9}$, los problemas de salud mental como consecuencia de la guerra $\operatorname{civil}^{10}$ y el consumo de drogas inyectables relacionadas con el uso al VIH. ${ }^{11}$ En Guatemala la Encuesta Nacional DUSI, en población adolescentes de nivel básico y diversificado de educación, sector publico de 1998, es uno de los primeros estudios que SECCATID impulso. El Estudio Nacional de Desregulación Psicológica y su relación con el consumo de drogas en jóvenes adolescentes guatemaltecos de 2003 es un enfoque interesante a los problemas asociados al consumo. ${ }^{12-15}$

Datos reportados en la tesis "Perfil inicial en salud mental del guatemalteco"16 indican que de 3,119 entrevistados el $7.6 \%$ de la población ha padecido algún TSM. El 8.3\% reportó tener familiares con TSM, el $10.48 \%$ indicó que consume tabaco, el $8.28 \%$ ingiere alcohol y un $34 \%$ que consume algún tipo de drogas. En el Informe sobre los sistemas de salud mental en Guatemala, de la OMS, en $2006^{17}$ reporta que los centros de atención ambulatoria $29 \%$ consultan por trastornos del ánimo, 29\% por trastornos neuróticos, esquizofrenia $17 \%, 3 \%$ por abuso de sustancias y $20 \%$ por otros trastornos. La prevalencia de TSM en la población guatemalteca es de $27.8 \%$. La Encuesta Nacional de Salud Mental (ENSM), del $2009^{18}$ en una población de 7.139 .575 personas reporta una prevalencia de TSM de $7.8 \%$ de trastornos mentales y del comportamiento debidos al consumo de sustancias psicotropas, de estas el $5.2 \%$ es por abuso/dependencia alcohólica, 3.7\% por abuso/dependencia de tabaco y $0.2 \%$ por abuso/dependencia drogas ilícitas. Trastornos depresivos reporta $6.4 \%$ y ansiedad generalizada $3.2 \%$. El TM mas frecuente es la depresión (6.4\%) seguida de la dependencia del alcohol (5.2\%).

En Estados Unidos se demostró que el 79\% de las personas diagnosticadas con TSM tenían más de un trastorno. Los resultados de la ENSM de Guatemala muestran que el $48 \%$ de la población total fue diagnosticada con más de un TSM. La prevalencia de comorbilidad es cada vez mas alta y requiere más investigación debido a que es complejo su diagnostico, especialmente por la dificultad de establecer cual de los dos trastornos es el que inicia primero y dispara el segundo. Para fines de esta investigación se definió la comorbilidad de distrés psicológico como un conjunto de síntomas y signos inespecíficos cuya severidad incrementa la probabilidad de que la persona sea portadora de una enfermedad psiquiátrica. Estos síntomas pueden ser leves, moderados o suficientemente severos como para afectar el funcionamiento individual en la esfera social, ocupacional, educativa, entre otros, y por ende requerirá de abordajes terapéuticos diferentes. La presencia de distrès psicológico severo aumenta en la persona la probabilidad de presentar ansiedad y depresión facilitando la detección y el seguimiento de estos cuadros.

El objetivo de este estudio es determinar la prevalencia de la comorbilidad entre el distrés psicológico y el abuso de drogas en pacientes en tratamiento en dos centros de la ciudad de Guatemala, el Centro de Tratamiento Ambulatorio de SECCATID y el Cas Hogar Jireh. La duración de la recolección de datos comprende de septiembre a octubre de 2010. Esta investigación presenta datos de las variables socio demográficas, antecedentes judiciales, resultados de distrès psicológico, percepción de funcionalidad familiar de los encuestados, y el nivel de satisfacción con la atención que se les brinda en los centros de tratamiento. 


\section{METODOLOGÍA}

Esta investigación es parte de un estudio epidemiológico descriptivo de prevalencia, multicéntrico, de corte transversal, en población de mayores de 18 años, que está en tratamiento por abuso/dependencia de drogas, que se realizó en Brasil, Chile, Guatemala, Jamaica, Nicaragua, Panamá, Paraguay y Uruguay.

Los dos centros seleccionados para la investigación están ubicados dentro del área urbana en la ciudad de Guatemala. Para la recolección de datos se requirió primero de un proceso de formación a cargo de la investigadora principal al equipo de tres investigadores asistentes. Se realizo una prueba piloto para validar los instrumentos y ajustar vocabulario y contextualizarlo al país. Los pacientes que contestaron en el piloto quedaron fuera de los datos del estudio que se presenta en este artículo. Se seleccionaron pacientes voluntarios para participar del Centro de Tratamiento Ambulatorio de SECCATID y la Casa Hogar Jireh quienes por escrito extendieron su consentimiento de participación.

Se observo la dinámica de trabajo y de atención en ambos centros durante más de un mes. Luego de establecida la mecánica de ingreso y tiempo de estancia en cada uno se informo de la investigación que se realizaría a partir del mes de agosto. Las autoridades permitieron reuniones abiertas con los pacientes para explicar el sistema de recolección de datos y formar a los investigadores asistentes. La muestra calculada fue de 93 personas. La recolección de datos se hizo de septiembre a octubre de 2010 contando con la participación de personal del equipo de psicólogos, en SECCATID y supervisores Jireh.

Se tomo en cuenta a toda la población que llegaba a tratamiento durante el tiempo de levantado de datos y los que residían en el centro al momento de aplicar el cuestionario, se invito a través de información verbal a todos a participar voluntariamente en llenar el cuestionario. Los menores de 18 años fueron excluidos así como los que no deseaban firmar el consentimiento informado. Los pacientes que estaban en otras actividades del Centro y no quisieron participar también fueron excluidos de la muestra. En la Casa Hogar Jireh de 75 pacientes residentes y que ingresaron al centro durante el tiempo del levantado de datos, 61 participaron voluntariamente.

Del Centro de Tratamiento Ambulatorio de 33 pacientes que llegaron a tratamiento durante el tiempo de recolección de datos, 30 aceptaron llenar el consentimiento informado. Dos pacientes que llenaron el cuestionario fueron eliminados al terminar el cuestionario porque solicitaron retirarse del estudio. Quedo una muestra de 91 personas. Se utilizaron tres instrumentos con los permisos debidos, fueron autoadministrados y en el caso de una persona analfabeta se realizo entrevista. Se utilizo el cuestionario EULAC-CICAD de 30 preguntas socio-demográficas, historia de abuso de drogas y alcohol, antecedentes judiciales, problemas mentales pasados y actuales, y datos del Centro de tratamiento el segundo instrumento es el cuestionario Kessler-10 (K-10) de diez preguntas que mide el distrès psicológico ${ }^{19} \mathrm{y}$ según puntaje lo clasifica en distress leve (de 10 a 15 puntos), moderado (16-21), severo (22-29) y muy severo (30-50).

Está demostrado que un nivel muy alto de distrés psicológico obtenido a través de la puntuación de la escala K-10 está asociada con una alta probabilidad de tener un trastorno de ansiedad o depresión. ${ }^{20}$ Como otros estudios en este caso se trabajaron las dos ultimas categorías, distress severo y muy severo juntos en el análisis. El cuestionario APGAR-familiar ${ }^{21}$ estima la percepción de funcionalidad de la familia desde la perspectiva del usuario. Los valores de la escala van de cero a 10 y valora cinco aspectos de la función de la familia: la adaptabilidad, asociación, crecimiento, afecto y resolución de conflictos. Una puntuación total de siete o más sugiere una percepción de alta funcionalidad familiar; entre cuatro y seis, moderada disfunción familiar, y tres o menos, una severa disfunción familiar. Para este estudio, la prevalencia de la población de pacientes que reporta distrés psicológico severo y muy severo, representan la proporción de casos con comorbilidad, y se presenta como la más importante medida de frecuencia epidemiológica.

Los consentimientos informados, los formularios y cuestionarios respondidos fueron guardados en una caja de seguridad por la investigadora principal. Se utilizó el programa Epi Info versión 3.5.1 22 para introducir y analizar los datos. Para el análisis preliminar de datos fueron calculadas las proporciones de variables dicotómicas y otras categóricas (prevalencia). Para las variables numéricas fueron calculadas las medidas de tendencia central y de dispersión.

El estudio fue aprobado por la Oficina de Ética en Investigación del Centro de Adicciones y Salud Mental de Canadá (CAMH) Protocol Re- 
ference \# 225/2008 y por el Comité de Ética del Ministerio de Salud Publica y Asistencia Social de Guatemala.

\section{RESULTADOS}

La tabla 1 muestra las características socio demográfica de la población encuestada. La mayoría de los encuestados eran hombres, 95.6\% (87 hombres y cuatro mujeres); el grupo etáreo que predomino es el de 24 a 39 años, $8.35 \%(n=44)$ La edad máxima reportada es de 70 años. Hubo pocos adultos jóvenes 19.78\%. El 93.41\% de los encuestados eran guatemaltecos, un $4.40 \%$ extranjeros. La categoría más frecuente de autodenominación de color/raza fue el mixto con $54.95 \%$ y seguido del blanco con $31.87 \%$, nativo $6.59 \%$ y afrodescendiente $5.49 \%$.

Tabla 1 - Características socio-demográficas de pacientes atendidos en Centros de tratamiento de abuso/dependencia de alcohol y drogas en la Ciudad de Guatemala, 2010

\begin{tabular}{llrr}
\hline \multicolumn{1}{c}{ Variable } & \multicolumn{1}{c}{ Categoría } & n & \multicolumn{1}{c}{$\%$} \\
\hline \multirow{2}{*}{ Sexo } & Femenino & 4 & 4.4 \\
& Masculino & 87 & 95.6 \\
Edad & $18-23$ & 18 & 19.78 \\
& $24-39$ & 44 & 48.35 \\
Nacionalidad & $40-+$ & 29 & 31.87 \\
& Nacional & 85 & 93.41 \\
& Extranjero & 6 & 6.59 \\
Raza/grupo étnico & Blanco & 30 & 32.97 \\
& Mixto & 50 & 54.95 \\
& Afrodescendiente & 5 & 5.49 \\
& Nativo & 6 & 6.59 \\
\hline
\end{tabular}

Otros datos importantes de resaltar (no mostrados en la tabla) son el estado civil, $47.3 \%$ son soltero, casados $20.9 \%$, en unión estable $19.8 \%$, separados $6.6 \%$, viudos $3.3 \%$, divorciados $1.1 \%$. Respecto a donde vivió en los últimos 30 días antes de ingresar al Centro de tratamiento el $44 \%$ refiere haber vivido con la familia de origen y un 5.5\% refiere haber vivido en la calle, reportaron haber vivido en casa del paciente ya fuera propia o alquilada $18.7 \%$ en ambos casos, $4.4 \%$ indicaron haber vivido en asilos, casas de acogida, refugios, $4.4 \%$ tuvieron otros lugares no especificados.

Respecto a donde han vivido antes de ingresar al centro el mayor porcentaje reporta con la madre en un $39.7 \%, 27.5 \%$ con la esposa, con el padre $18.7 \%$, con hermano/hermana un $16.5 \%$ y solos $6.6 \%$, con otro familiar un $5.5 \%$, menores porcentajes registraron madrastra, padrastro, novio/a, amigo/a. Saben leer y escribir $98.9 \%$ de la población encuestada. Respecto al nivel educacional, el $15.4 \%$ curso primaria completa, $14.3 \%$ primaria incompleta, $27.5 \%$ curso el nivel secundario completo, $23.1 \%$ secundario incompleto, Universidad completa $4.4 \%$ y $14.3 \%$ universidad incompleta, es de resaltar que $0.0 \%$ no asistió a la escuela. La relación laboral indica que $51.9 \%$ trabaja, $15.4 \%$ estudia y trabaja, $13.2 \%$ son estudiantes, $3.3 \%$ desempleados, $3.3 \%$ pensionados, $3.3 \%$ tareas de de casa, $1.1 \%$ no trabaja por discapacidad.

La tabla 2 muestra la principal o primera droga, licitas e ilícitas, identificada por importancia y por la cual reciben tratamiento. La pasta base de cocaína es referida como la primera droga $36.3 \%$, la pasta base en un segundo lugar $27.5 \%$ y la cocaína en polvo $16.5 \%$ en tercer lugar, con porcentajes menores la marihuana $10.9 \%$, el alcohol 5.4\% y los opioides 3.3\%. No reportaron ninguna otra droga como los estimulantes, los hipnóticos y sedativos, alucinógenos, inhalantes y abuso de medicación recetada por médico ni esteroides anabólicos.

Tabla 2 - Primer droga identificada por la población según importancia, por la que actualmente reciben tratamiento en dos Centros de tratamiento en la ciudad de Guatemala. Septiembre-octubre 2010

\begin{tabular}{lrr}
\hline \multicolumn{1}{c}{ Tipo de droga } & n & \% \\
\hline Alcohol & 5 & 5.4 \\
Marihuana & 10 & 10.9 \\
Cocaina & & \\
Pasta base & 25 & 27.5 \\
Polvo & 15 & 16.5 \\
Crack & 33 & 36.3 \\
Opioides & 3 & 3.3 \\
\hline
\end{tabular}

En lo que respecta a las drogas, licitas e ilícitas, consumidas durante los últimos 30 días antes de iniciar tratamiento, la tabla 3 describe la distribución donde el alcohol resalta con $78 \%$, cannabis con $37.4 \%$, crack con $36.3 \%$, cocaína $27.5 \%$ y en menores porcentajes inhalables $14.3 \%$, abuso de medicación prescrita $6.6 \%$ y esteroides anabólicos $4.4 \%$ al igual que la LSD $4.4 \%$ y otros alucinógenos $4.4 \%$. Otras drogas aparecen con porcentajes mas bajos. 
Tabla 3 - Distribución de las drogas consumidas durante los últimos 30 días antes de iniciar el tratamiento de los pacientes atendidos en Centros de tratamiento de la Ciudad de Guatemala. Septiembre-octubre 2010

\begin{tabular}{lrr}
\hline Categoría & $\mathbf{n}$ & \% \\
\hline Abuso de medicación prescrita & 6 & 6.6 \\
Alcohol & 71 & 78 \\
Anfetaminas (uso no prescrito) & 2 & 2.2 \\
Benzodiacepinas & 5 & 5.5 \\
Cannabis (marihuana, ganja y otros derivados) & 34 & 37.4 \\
Cocaína & 25 & 27.5 \\
Crack & 33 & 36.3 \\
Esteroides anabólicos & 4 & 4.4 \\
Heroína & 1 & 1.1 \\
Inhalables & 13 & 14.3 \\
LSD & 4 & 4.4 \\
Metadona & - & - \\
Metanfetaminas (éxtasis) y otros derivados & 3 & 3.3 \\
Opioides & 3.3 \\
Otros alucinógenos & 4 & 3.4 \\
Pasta de coca (basuco, paco) & 3 & 3.3 \\
Barbitúricos & 1 & \\
\hline
\end{tabular}

La tabla 4 muestra el comportamiento de la variable relacionada con la coexistencia de otras patologías psiquiatritas. Solamente $2.4 \%$ reporta como diagnostico actual ansiedad, $3.3 \%$ por depresión, el $4.4 \%$ por esquizofrenia y $4.4 \%$ por trastorno bipolar. Resaltan los porcentajes más altos asociados a esta variable en la columna No sabe y la columna No le sigue en segundo lugar. La mayoría de los pacientes reportan no tener actualmente un diagnostico de salud mental.

Tabla 4 - Coexistencia de otras patologías psiquiátricas en usuarios que consumen drogas y están actualmente en tratamiento en dos Centros de la ciudad de Guatemala. Septiembre-octubre 2010

\begin{tabular}{|c|c|c|c|c|c|c|c|c|c|}
\hline \multirow{2}{*}{ Variable } & \multirow{2}{*}{ Categoría } & \multicolumn{2}{|c|}{ Si } & \multicolumn{2}{|c|}{ No } & \multicolumn{2}{|c|}{ No sabe } & \multicolumn{2}{|c|}{ NR } \\
\hline & & $n$ & $\%$ & $n$ & $\%$ & $\mathbf{n}$ & $\%$ & $\mathbf{n}$ & $\%$ \\
\hline \multirow{5}{*}{$\begin{array}{l}\text { Diagnóstico actual de } \\
\text { salud mental }\end{array}$} & Ansiedad & 2 & 2.4 & 32 & 35.2 & 50 & 54.9 & 7 & 7.6 \\
\hline & Depresión & 3 & 3.3 & 16 & 17.6 & 61 & 67.0 & 11 & 12.1 \\
\hline & Esquizofrenia & 4 & 4.4 & 2 & 2.2 & 67 & 73.6 & 18 & 19.7 \\
\hline & $\begin{array}{l}\text { Transtorno } \\
\text { bipolar }\end{array}$ & 4 & 4.4 & 1 & 1.1 & 66 & 72.5 & 20 & 21.9 \\
\hline & Otros & 3 & 100 & - & - & - & - & - & - \\
\hline
\end{tabular}

Los datos que muestra la tabla 5 se refieren a las pruebas K-10 que se utilizo para valorar el distrés psicológico y APGAR-familiar que se aplico para establecer la percepción individual de la funcionalidad familiar de los pacientes. El K-10 reporta distrés bajo en un $8.79 \%$ de la población estudiada, un $19.78 \%$ moderado, $31.87 \%$ severo y $39.56 \%$ muy severo. Los datos relevantes para este estudio son los de distrés severo y muy severo debido a que se relaciona significativamente con diagnósticos de ansiedad y depresión. Los valores de las puntuaciones de la escala K-10 de distrés psicológico variaron entre ocho y 50 (promedio=27.70; mediana $=27$ ).

La distribución indica que, en media, los pacientes tienen niveles de distrés considerados en la franja de "severos" (entre 22 y 29). La prevalencia de distrés severo y muy severo es de $71.43 \%$ $(n=65)$ en los pacientes con trastorno por consumo de drogas licitas e ilícitas que fueron atendidas en dos centros de la ciudad de Guatemala.

Los valores de las puntuaciones de la escala K-10 de distrés psicológico variaron entre ocho y 50 (promedio=27.70; mediana=27). La distribución indica que, en media, los pacientes tienen niveles de distrés considerados en la franja de "severos" (entre 22 y 29).

Los resultados del APGAR-familiar reportan que el $59.34 \%$ de los pacientes no perciben disfunción en sus familias, el 23,07\% refiere moderada disfuncionalidad y 17.59 encuentra severamente disfuncional su familia. 
Tabla 5 - Grados de distrés psicológico según K-10 y niveles de percepción de funcionalidad familiar APGAR-familiar en los usuarios con trastorno por consumo de sustancias en tratamiento en la Ciudad de Guatemala de septiembre a octubre 2010

\begin{tabular}{llrc}
\hline Variable & Categoría & Si & $\%$ \\
\hline \multirow{2}{*}{ Distrés psicológico } & Bajo (10-15) & 8 & 8.79 \\
(escala K-10) & Moderado (16-21) & 18 & 19.78 \\
& Severo (22-29) & 29 & 31.87 \\
Funcionalidad familiar percibida & Muy severo (30-50) & 36 & 39.56 \\
(APGAR-família) & Mormofuncional (>7) & 54 & 59.34 \\
& Moderadamente disfuncional (4-6) & 21 & 23.07 \\
& Severamente disfuncional $(<3)$ & 16 & 17.59 \\
\hline
\end{tabular}

\section{DISCUSIÓN}

El escenario mundial de salud mental enfatiza la importancia de atender y estudiar la comorbilidad entre trastornos de salud mental y trastornos por uso de sustancias ${ }^{23}$ no solo por lo frecuente que se ha determinado que es, pues tiene una prevalencia del $50 \%$ según estudios realizados, sino por las repercusiones que tiene en el desarrollo de un país. ${ }^{24}$ Los factores asociados a la comorbilidad entre estos dos trastornos son múltiples pero deben destacarse las características individuales, los sociales, familiares y el tipo de drogas que se consumen. En este estudio los pacientes 87 , son hombres, y cuatro son mujeres, esto se debe a que en uno de los centros encuestados solo brindan atención a hombres. La mayoría tienen bajos recursos asociada a una historia de uso y dependencia de alcohol. En cuanto a la edad son predominantemente jóvenes adultos entre 24 y 39 un $48.35 \%$ y $31.87 \%$ son mayores de 40 años. Se perciben en su mayoría como etnia mixta (ladinos conformados por mestizos y descendientes de criollos) lo que responde a la realidad de más del $60 \%$ de la población guatemalteca.

Las variables educativa y laboral reportan condiciones protectoras para la mayoría de la población objeto de este estudio pues favorecen la inclusión social. A nivel educativa presentan escolaridad de nivel secundario completo e incompleto el $50.6 \%$ dato que se asocia en el porcentaje del $51 \%$ que trabaja como empleado o de forma independiente a pesar de los indicadores de desempleo del país. El $47.3 \%$ son solteros favoreciendo así que el $44 \%$ viva con la familia de origen según reporta como lugar de vivienda antes de ingresar al centro de tratamiento y conviviendo con la madre en un $39.7 \%$ y con la esposa un $27.5 \%$. Mas de la mitad de los encuestados refiere haber tenido problemas con la ley relacionados con el consumo de sustancias durante su vida.

A pesar de que $78 \%$ indican haber consumido alcohol 30 días antes de ingresar al centro de tratamiento, la droga por la que buscaron tratamiento el $36.3 \%$ de los encuestados es el crack, $27.5 \%$ por la pasta base y por 16.5 el polvo de cocaína. Estos datos no corresponde con la $1^{\circ}$ Encuesta Continua de hogares acerca del consumo de sustancias adictivas realizada en el año 2007 conjuntamente por SECCATID, Instituto Nacional de Estadística, Sección de Asuntos Narcóticos (NAS) - Embajada de los Estados Unidos de América CICAD, donde se establece que el alcohol con el 52.0\% representa la droga mas consumida.

Con respecto al contexto familiar es importante destacar que el $59.34 .0 \%$ percibe la normofuncionalidad familiar esto puede deberse a que el consumidor se ve a si mismo como un problema y sobre el recaen las dinámicas complejas producto de su adicción y el resto de la familia gira en torno a esa problemática. Es importante destacar la baja prevalencia de psicopatológicas coexistentes actualmente indicadas por los pacientes. Esto puede deberse a que no se les hace un diagnostico de salud mental al llegar al centro de tratamiento, sino que se les ingresa por el trastorno de uso de sustancias y por el desconocimiento del diagnóstico que de el se tiene en el centro de tratamiento. También puede deberse a que existe baja atención a las patologías psiquiatritas coexistentes en los centros que participaron en este estudio.

Finalmente la presencia de distrés psicológico en personas con uso y abuso de alcohol y drogas es la frecuencia epidemiológica más relevante de este estudio. Con el cuestionario que se aplico se busco identificar la comorbilidad entre los trastornos de salud mental y otros trastornos por el uso de sustancias. El K-10 es útil para responder a la pregunta: “¿Hay evidencia de un posible problema de salud mental que requiera una mayor investigación en esta población?". ${ }^{25}$ La respuesta para nuestro estudio es que si. La escala de Kessler, K-10 permite establecer la presencia de distrés psicológico en la población seleccionada ya que un $71.43 \%(n=65)$ reporta distrés severo y muy severo lo que indica alto riesgo de ansiedad y depresión. 


\section{CONCLUSIONES}

Sobre la base de los objetivos propuestos para este estudio se puede concluir que en los dos centros de tratamiento, SECCATID y Casa Hogar Jireh, durante los meses de septiembre y octubre de 2010 en la ciudad de Guatemala, la prevalencia de distrés psicológico severo y muy severo fue de $71.43 \%$ lo que permite identificar el riesgo de trastornos mentales, de ansiedad y depresión en este grupo poblacional. Estos hallazgos confirman los datos reportados por otros estudios de la existencia de alto riesgo de trastornos de salud mental en personas que usan y abusan del consumo de sustancias. Los factores individuales, sociales, familiares y legales inciden en el uso de crack, pasta base y polvo como drogas de primera droga identificada por la población estudiada. El grupo de usuarios percibe una alta prevalencia de normofuncionalidad familiar. Un bajo porcentaje de los pacientes de estos centros recibe atención a la comorbilidad.

\section{Limitaciones}

Las limitaciones que presenta este estudio están relacionas con el tipo de estudio, la selección de los centros de tratamiento y los instrumentos. El diseño del estudio, de corte transversal, limita los datos a un momento de la vida de los pacientes y se pierde la dinámica general de vida en los centros de atención. La selección de los lugares del estudio por las características propias de cada uno en estructura y organización presentan diferencias en la población que atienden. Uno de los centros atiende solo hombres el otro de ambos sexos, uno privado otro público, siendo aspectos que determinan el perfil del usuario con el que se trabajo en esta investigación. Esto limita la generalización de resultados a la población en general que usan y abusan sustancias. El instrumento autoaplicado para recolectar datos fue una limitante por los datos faltantes.

\section{Recomendaciones}

Capacitar a los profesionales de los centros de atención de alcohol y drogas sobre la prevalencia de distrés psicológico en los diferentes niveles.

Capacitar a los profesionales de los centros de atención de alcohol y drogas sobre la existencia, frecuencia y relevancia de la comorbilidad.

Implementar en los centros de atención de alcohol y drogas el uso de screening para detectar presencia de trastornos mentales coexistentes para la pronta identificación de comorbilidad.
Continuar estudios de aplicación del K-10 para validad en grupos poblacionales diversos $\mathrm{y}$ establecer las diferencias.

Agregar en los diseños curriculares de pregrado y posgrado de ciencias de la salud temas y contenidos sobre comorbilidad psiquiatrita y la importancia de identificación la misma en el tratamiento para el abuso y dependencia de drogas.

Evaluar los centros de tratamiento para establecer la capacidad de manejo de pacientes con comorbilidad.

Hacer planes de mejora para los centros de tratamiento de abuso y dependencia de drogas para atención de trastornos mentales.

Realizar otros estudios de seguimiento a este para profundizar en el distrés psicológico y comorbilidad en otras poblaciones.

\section{AGRADECIMIENTOS}

Este estudio se realizo gracias al apoyo, asesorìa y patrocinio del Gobierno de Canadá, de la Comisión Interamericana para el Control del Abuso de Drogas (CICAD) de la OEA y al Centro de Adicciones y Salud Mental (CAMH) de Canadá, Universidad de Toronto, Universidad de San Carlos de Guatemala, Facultad de Ciencias Medicas; Ministerio de Salud Publica y Asistencia Social de Guatemala, SECCATID, Centro de Tratamiento y sus profesionales de salud, Casa Hogar Jireh y sus supervisores y especialmente a los pacientes que aceptaron participar en este estudio.

\section{REFERENCIAS}

1. Evans K, Sullivan JM. Dual diagnosis. counseling the mentally III substance abuser. New York (US): The Guilford Press; 2001.

2. Dalex DC, Moss HB. Dual disorders. Counseling clients with chemical dependency and mental illness. $3^{\mathrm{a}}$ ed. Minnesota (US): Hazelden Foundation Center; 2002.

3. Kessler RC. Epidemiology of psychiatric comorbidity. In: Tsuang MT, Tohen M, Zahner GEP, editors. Psychiatric epidemiology. New York (US): WileyLiss; 1995. p. 179-97.

4. Grant BF. The influence of comorbid major depression and substance use disorders on alcohol and drug treatment: results of a national survey. In: National Institute on Drug Abuse (NIDA). Treatment of drug-dependent individuals with comorbid mental disorders. Rockville (US): NIH; 1997.

5. World Health Organization (WHO). Mental health gap action programme (mhGAP). Washington (US): 
WHO; [accesso 2009 Jun 25] Disponible en: http:/ / www.who.int/mental_health/mhgap/en/

6. Junta Internacional de Fiscalización de Estupefacientes, Oraganización de las Naciones Unidas. Informe de la Junta Internacional de Fiscalización de correspondiente a 2003. JIFE, ONU; 2003. [accesso 2009 Jun 25]. JIFE-ONU Disponible en: http://www.incb.org/incb/es/ annual_report_2003.html

7. Organización de los Estados Americanos, Comisión Interamericana para el Control del Abuso de Drogas. Mecanismo de evaluación multilateral: Guatemala: evaluación del progreso de control de drogas 20052006. Guatemala: OEA,CICAD; 2006.

8. World Health Organization (WHO). Guatemala menthal health atlas. Washington (US): WHO; 2005 [accesso 2009 Jun 25] Disponible: http://www. who.int/mental_health/evidence/mhatlas05/en/ index.html

9. Kanteres F, Lachenmeier DW, Rehm J. Alcohol in Mayan Guatemala: consumption, distribution, production and composition of cuxa. Addiction. 2009 May; 104(5):752-9.

10. Sabin M, Sabin K, Kim HY, Vergara M, Varese L. The mental health status of Mayan refugees after repatriation to Guatemala. Rev Panam Salud Publica. 2006 Mar; 19(3):163-71.

11. Shehane E, Jacobdon J, Iguchi M. Un estudio para identificar comportamientos de riesgo y necesidades de prevención de VIH/SIDA en Centros de Tratamiento de Drogadicción en la Ciudad de Guatemala y San Salvador: proyecto para establecer tratamiento, rehabilitación y red de reinserción social en Centroamérica (CAM H90). Guatemala: Universidad del Valle de Guatemala, Oficina de Naciones Unidas contra las Drogas y el Delito; 2008[accesso 2009 Jun 25]. Available at http:/ / www. seguridad.gob.sv/observatorio/demanda/2008/ VIH_UDI_Esp_Final.pdf

12. Secretaria Ejecutiva Comisión contra las adicciones y el tráfico ilícito de drogas. Encuesta Nacional DUSI, en población adolescente del nivel básico y diversificado de educación, sector publico. Guatemala: Universidad Johns Hopkins; 1998.

13. Sistema Interamericano de datos uniformes sobre consumo de drogas. Encuesta de estudiantes de enseñanza media, consumo de drogas en estudiantes. Guatemala: SIDUC/CICAD; 2003.

14. Secretaria Ejecutiva Comisión contra las adicciones y el tráfico ilícito de drogas, Embajada de Estados
Unidos de América en Guatemala. Estudio nacional de desregulación psicológica y su relación con el consumo de drogas en jóvenes adolescentes guatemaltecos: principales hallazgos. Guatemala: SECCATID; 2003.

15. SECCATID/CICAD/NAS/INE. Primera encuesta Nacional en hogares sobre salud, consumo de alcohol, tabaco y otras sustancias adictivas. Guatemala: SECCATID/CICAD/NAS/INE; 2007.

16. Robles GLL. Perfil inicial en Salud Mental del Guatemalteco [tesis]. Guatemala: Universidad de San Carlos de Guatemala, Escuela de Ciencias Psicológicas; 2006.

17. Organización Mundial de la Salud. Instrumentos de evaluación para sistemas de salud mental: informe sobre el sistema de salud mental en Guatemala. Guatemala: IESM/OMS; 2006.

18. Universidad de San Carlos de Guatemala. Encuesta Nacional de Salud Mental, del 2009: descripción epidemiológica de trastornos de salud mental en hombres y mujeres de 18 a 65 años de edad realizada en la República de Guatemala. Guatemala: Universidad de San Carlos de Guatemala; 2009.

19. Kessler RC, Andrews G, Colpe LJ, Hiripi E, Mroczek DK, Normand SLT, et al. Short screening scales to monitor population prevalances and trends in nonspecific psychological distress. Psychol Med. 2002 Aug; 32(6):959-76.

20. Andrews G, Slade T. Interpreting scores on the Kessler Psychological Distress Scale (K10). Aust N Z J Public Health. 2001 Dec; 25(6):494-7.

21. Smilkstein G. The family APGAR: a proposal for family function test and its use by physicians. J Fam Prac.1978 Jun; 6(6):1231-9.

22. Centers for Disease Control and Prevention. Epi Info version 3.5.1. 2008 [access 2009 Jun 25]. http:/ / wwwn.cdc.gov/epiinfo/

23. Rush B. Editorial. Int J Ment Health Addiction. 2008 Jan; 6(1):1-7.

24. Saraceno B, Saxena S, Caraveo-Adnurga J, Khon R, Levav I, Caldas de Almeida M. Los trastornos mentales en América Latina y el Caribe: asunto prioritario para la Salud Publica. Rev Panam Salud Publica. 2005 Nov; 18(4-5):229-40.

25. Zimmerman M. To screen or not to screen: conceptual issues in screening for psychiatric disorders in psychiatric patients with a focus on the performance of the psychiatric diagnostic screening. Int J Ment Health Addiction. 2008 Jan; 6(1):53-63. 